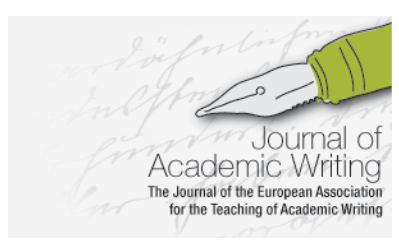

Journal of Academic Writing

Vol. 2 No. 1 Autumn 2012, pages 59-73

\title{
Contrastive Genre Mapping in Academic Contexts: an Intercultural Approach
}

\author{
Otto Kruse \\ Zurich University of Applied Sciences, Switzerland \\ Madalina Chitez \\ Zurich University of Applied Sciences, Switzerland
}

\begin{abstract}
This paper presents a comparison of genre use at three Swiss universities from different language regions. The methodology is one of contrastive genre mapping in which we connect two lines of research usually seen as distinct approaches. The aim of the study is to find ways of comparing the writing cultures of different languages by collecting and comparing the genres used for teaching. Data about genres were gathered through questionnaires in which students and faculty members were asked to describe writing assignments and student texts. From the answers to these questionnaires, genre inventories were constructed and then re-checked with insiders in faculty discussions or interviews. As results, lists of genres from the individual universities are presented, as are the patterns of genre families into which the genres were classified. It turned out that genre use shows strong similarities across the three universities. The main genre families are presented and differences between universities are discussed.
\end{abstract}

\section{Introduction}

The aim of this paper is to describe and discuss contrastive genre mapping in academic contexts. We hope to come to a better understanding of what writing cultures are and what methods might be applied for their study. We report on a project in which we compared three Swiss Universities of Teacher Education (UTE) sharing a similar organizational structure but using different languages for teaching (French, German and Italian). We used a contrastive methodology to find out what language-specific differences may exist. Our approach is located at the crossroads of intercultural writing research (for instance, Connor, Nagelhout, and Rozycki 2008, and Foster and Russell 2002) and genre mapping or genre analysis (for instance, Bhatia 2002, and Nesi et al. 2008), two lines of research approaching genre from different perspectives. From the former (contrastive research), we preserve the focus on the influence that writing cultures have on individual text production. From the latter (genre mapping/ analysis) we keep the focus on collecting, inventorying and analyzing genres.

The reason for such a study is an almost complete lack of knowledge about how differently writing and genre use are practiced in the main language regions of Switzerland. It is not known to what extent academic writing follows a Swiss model and how much it is connected with the neighboring cultures of France, Germany and Italy ${ }^{2}$, each using one of the three Swiss national languages. Though we will not be able to answer this question conclusively, we will provide a theoretical and methodological base for approaching it.

\footnotetext{
${ }^{1}$ This study was funded by the Swiss State Secretariat for Education and Research (Project No.: C08.0121). It was also part of COST-Action IS0703 'European Research Network on Learning to Write Effectively' (http://www.cost-Iwe.eu/).

${ }^{2}$ The fourth Swiss language, Romansh, is not considered here as it is not used in university teaching.
} 
There were several strategic considerations guiding us through the process of contrastive genre mapping. In the first place, our focus was on writing cultures in institutions rather than on individual writing. ${ }^{3}$ We believe that writing cultures are influenced by the higher order structures connected with languages and educational systems and determine, in turn, the lower order issues of writing practices, course organization and genre use. A second strategic decision was the use of questionnaires instead of text analysis or corpus methods as Nesi et al. (2008) have used for genre mapping. Before we move into a detailed comparison of linguistic aspects of genres in three languages, we argue, it is necessary to know which genres there are. We had every reason to believe, that the number of genres used in tertiary education was much higher than is generally assumed, but we did not know whether we could expect similar genres in French, German, and Italian teaching.

To come to valid comparisons, we had to identify similar disciplines in the three language regions. One of the disciplines that exists in all three major Swiss language regions is teacher training at the Universities of Teacher Education. Data were collected from the following universities:

- HEP-BEJUNE : Haute école pédagogique des cantons de Berne, du Jura et de Neuchâtel, in which French is the teaching language

- $\quad$ PH Zürich: Pädagogische Hochschule Zürich, in which German is used as a teaching language

- SUPSI-DFA: Scuola universitaria professionale della Svizzera italiana - Dipartimento formazione e apprendimento, from Locarno $(\mathrm{TI})$, where courses are held in Italian.

The three universities offer similar but not identical courses. Chosen for this study where BA and MA programs in Primary and Secondary Education (but not Preschool Education, which is offered as well). Differences exist, for instance, at the level of the secondary education programs, which are offered as BA plus MA programs at PH Zurich and HEP-Bejune, whereas, at SUPSI-DFA, BA programs cover Primary Education only while all MA students in Secondary Education need a BA degree from another university. BA degrees at SUPSI cover Preschool and Primary Education only.

\section{Method}

\subsection{Genre mapping}

The term 'genre mapping' is fairly common in Cultural Studies when it comes to classifying films or pieces of music and literature. In Rhetorical Studies it was first used in the context of Systemic Functional Linguistics to describe genre use and genre systems in primary and secondary education (Custance 2006 and Martin and Rose 2008). The intention of this procedure is to understand which genres students have to write and how writing development can be influenced by genre teaching. Australian schools use genre mapping to provide teachers with a whole-school genre map', a list of genres to be used in teaching. An extensive example of genre mapping in school is provided by Martin and Rose (2008) even though they avoid the term 'genre mapping' and speak of 'mapping culture'. They describe school genres under the headings of five 'genre families': stories, histories, reports and explanations, procedures, and procedural accounts. The use of a fixed classification scheme as a starting point for mapping, however, seems questionable. For university purposes, a more open procedure should be preferred.

We consider genre mapping as a systematic procedure that helps detecting, collecting, defining and understanding genres in a certain professional, academic or cultural field. Genre mapping leads to inventories of genres, often connected with the collection of genre examples which then may be grouped, classified and put in a systematic order. Genre mapping usually has to make use of insider information and needs several analytic/interpretive steps to come to an understanding of how genres are structured, used and functionally connected to context. We see genre mapping as a basis for indepth studies of texts as in text analytic or corpus approaches.

An example of genre mapping research in higher education that avoids a priori classifications is provided by Nesi et al. (2008), who collected student texts within three different UK universities from 33 departments and 627 student contributors. They classified the texts into more than one hundred genres and grouped them into thirteen genre families. In the latest publication (Nesi and Gardner 2012),

${ }^{3}$ For a more detailed description of writing cultures see Chitez and Kruse (2012). 
the thirteen genre families are explained in the framework of functional contexts like explaining, developing arguments, reporting research and preparing for professional life.

Genre mapping is a procedure that accounts for the fact that in almost any context or cultural field large numbers of interrelated genres are in use. We use the term 'genre inventory' when referring to the total of all (major) genres used in a certain context. 'Genre families' may be considered groups of genres sharing similarities in shape, register or purpose like the letter genres or the critique genres (Nesi et al. 2008). We speak of 'genre systems' (Bazerman 1994) when genres are functionally related to each other in a professional context like, for instance, the reporting genres in science communication (Swales 1990). Bhatia (2002) speaks of 'genre colonies' in the case of 'closely related and overlapping genres' as would be the case, for instance, with the textbook genres.

\subsection{Research steps}

Contrastive genre mapping is a sequential process carried out in several steps. The first step in our project consisted of data collection with the help of online questionnaires. The data collection was carried out by the universities themselves, after coordination meetings with university officials or research units of each university. Each of them received a link to the electronic questionnaire and a standard letter that was sent to their students and lecturers in November and December 2010. Questionnaires were administered with the help of a SPSS-supported online tool, which allowed the questionnaire to be used in five languages, and which automatically transformed the data into SPSS files.

The questionnaires are basically built on self-assessments of how competent students feel in different writing sub-skills and on judgements of what lecturers appreciate in their students' writing. Both questionnaires took no longer than ten minutes to be filled in. A total of 231 students (from 1009) and 84 lecturers (from 277) returned the questionnaire, which corresponds with response rates of $21.1 \%$ and $30.3 \%$, respectively.

The questionnaires are subdivided in several parts. The part evaluated in this paper deals with questions about genre use. Students were asked What was the last paper you had to write at University that was evaluated?' The rubric was divided into Name of the paper' and 'Short description of the type of text'. For the faculty questionnaire, the question was: 'If students write papers in your classes, what types of texts (genres) do they write? Please specify them or give a short description.'

In the second step, we organized workshops and panel discussions with faculty, to receive background information. If this was not possible, we conducted interviews with faculty members. The first workshop took place in February 2011 at the Pedagogical University of Zurich, the second in May 2011, at SUPSI, in Locarno. The questions that were discussed were: What writing skills should be developed at your university?; What types of text are used?; and How do writing competences develop? We also presented and discussed our first draft of the genre inventory. Interviews were taken at HEP-BEJUNE between June and August 2011. The questions asked were similar to the workshop questions.

In the third step, we grouped genres for each university into genre families. The classification was, in turn, made progressively:

- two genre lists per university (one for students and one for faculty) due to the fact that, in some cases, the students mentioned other genres than their teachers or that, in other cases, the same genres were named differently by the two categories of informants;

- $\quad$ one aggregate genre list per university combining both student and faculty answers, which was completed after discussions during the workshops and interviews clarifying genre synonymy and genre affiliation;

- a combined list of genre families for all three universities showing similarities and differences between the universities.

The classification led to a model of the genre system of Swiss UTEs indicating the main genres in their functional interrelations.

To understand the functional connections of genres with the educational setup of the institutions we also analyzed written materials from each of the universities. Instructions for student writing and regulations for thesis writing exist in all three schools. In this paper, we only refer to these materials as 
background information and don't provide a specific evaluation. The focus of our presentation is on the first steps to demonstrate how contrastive genre analysis arrives at descriptions of genre inventories and systems.

\section{Results}

\subsection{Preliminary genre inventories}

Information on genre use from the questionnaires led to heterogeneous lists of genre names which were used for a first tentative classification into genre families. For many genres, it turned out, there are no standard names and for some of them we received descriptions rather than precise labels. Some genre names include contextual information ('reflection on tutoring activities'), some referred to writing practices but not to genres ('portfolio based on the $12 \mathrm{PHZH}$ standards ).

Table 1: Genre inventory at PH Zurich - first classification (with literal translations).

\begin{tabular}{|c|c|}
\hline Genre family & Listed in survey as: \\
\hline Thesis & $\begin{array}{l}\text { - Didaktische Abschlussarbeit [didactic final paper] } \\
\text { - Master Thesis [master thesis] } \\
\text { - Vertiefungsarbeiten [in-depth study] }\end{array}$ \\
\hline Portfolio & $\begin{array}{l}\text { - Auf der Basis der } 12 \mathrm{PHZH} \text { Standards /Theoriearbeitsteil, } \\
\text { Praxisbeschreibung, eigene Interpretation und Konklusion [based on the } \\
12 \text { standards of } \mathrm{PHZH} / \text { theoretical part, practical part, interpretation and } \\
\text { conclusion] }\end{array}$ \\
\hline Lesson plan & $\begin{array}{l}\text { - Kommentierte Unterrichtsplanung [commented lesson plan] } \\
\text { - Unterrichtsplanung (mit Begründung, Material) [lesson plan] } \\
\text { - Prüfungsarbeit zu Didaktik des Bildnerischen Gestaltens Sek 1, } \\
\text { Unterrichtsplanung [exam paper on the methodology of visual arts, } \\
\text { - Secundary 1, lesson plan] } \\
\text { - Unterrichtsplanungen mit Begründungen des Vorgehens, Legitimation } \\
\text { des Unterrichts durch Zitieren von wissenschaftlichen Texten, Lehrplan } \\
\text { etc. [lesson plan with explanations on the procedure, legitimacy of the } \\
\text { lesson plans through quotations, teaching plan etc.] } \\
\text { - Didaktische Analysen [didactic analyses] } \\
\text { - Unterrichtsvorbereitungen [preparation for lessons] } \\
\text { - Fachdidaktische Planungsarbeiten mit didaktischen Analysen und } \\
\text { - Präparationen [didactic planning with didactic analyses and preparations] } \\
\text { - Sachanalysen, didaktische Analysen und Umsetzungsbeispiele für den } \\
\text { Unterricht/ Unterrichtsplanung [practical analyses, didactic analyses and } \\
\text { - applications examples for lesson/lesson plans] } \\
\text { - Unterrichtsprojekte [lesson projects] } \\
\text { - Planung von Lektionen [lesson planning] } \\
\text { - Lernprozessdokumentationen [learning process documentation] } \\
\text { theorye, theoriegestützte Konzepte für den künftigen Unterricht [personal } \\
\text { theoryported concepts of the future lessons] }\end{array}$ \\
\hline $\begin{array}{l}\text { Practical training } \\
\text { report }\end{array}$ & $\begin{array}{l}\text { - Falldokumentationen mit Kommentaren (kleinere Aufträge) [case studies } \\
\text { with comments (short papers)] }\end{array}$ \\
\hline Reflective text & $\begin{array}{l}\text { - Persönliche Reflexion [personal reflections] } \\
\text { - Persönliche Reflexion mit thematischer Vertiefung [personal reflection } \\
\text { with thematic immersion] } \\
\text { - Reflexive Texte [reflective texts] }\end{array}$ \\
\hline
\end{tabular}




\begin{tabular}{|c|c|}
\hline & $\begin{array}{l}\text { - Reflexionen zu eigener gestalterischer Praxis und zu individuellen } \\
\text { gestalterischen Projekten[reflection on personal creative practical } \\
\text { activities and on the individual creative projects] } \\
\text { - Reflexionen zu einzelnen Seminarveranstaltungen [reflections on } \\
\text { seminars] } \\
\text { - Learning journal: Students write a log on their teaching experiences re- } \\
\text { flecting on their experience and the planning process } \\
\text { - Reflexionstexte zum Mentorat [reflective texts on tutoring activities] }\end{array}$ \\
\hline Seminar paper & $\begin{array}{l}\text { - Literaturarbeit > Diskussion von wissenschaftlichen Grundlagentexten } \\
\text { [literature processing }>\text { discussion on basic scientific texts] } \\
\text { - Wissenschaftliche Texte [scientific texts] } \\
\text { - Leistungsnachweise, die einen theoretischen sowie einen praktischen Teil } \\
\text { beinhalten [performance records which include both theoretical and } \\
\text { practical aspects] } \\
\text { - Prüfungsarbeit mit Literaturrecherche, Cluster, didaktischer Analyse, } \\
\text { Grobplanung und exemplarischer Feinplanung [graded paper with } \\
\text { literature review, didactic analysis, rough planning and exemplary detailed } \\
\text { plan] } \\
\text { - Beschreibung und Erklärung von Experimenten bzw. Phänomenen. } \\
\text { Zusammenfassen des Hintergrundwissens [description and explanation of } \\
\text { experiments and phenomena. Summary of basic knowledge] } \\
\text { - Vergleichbar mit Seminararbeiten an der Universität. Basierend auf einer } \\
\text { eigenen Fragestellung/ These zu einem fachwissenschaftlichen oder } \\
\text { fachdidaktischen Thema [comparable with seminar papers at the } \\
\text { university. Based on a research question in the purely scientific or didactic } \\
\text { field] } \\
\text { - Geschichtsseminararbeiten (Miniforschungsarbeiten) [seminar paper in } \\
\text { history (mini research paper)] } \\
\text { - Fachwissenschaftliche Hausarbeit [scientific paper] } \\
\text { - Längere Arbeit - orientiert am Modell des wissenschaftlichen } \\
\text { Diskussionsbeitrages [elaborate paper - modelled on the scientific } \\
\text { debate] } \\
\text { - Zusammenfassung von Wissen aus der Literatur zum Thema Lernen und } \\
\text { Beschreibung deren Umsetzung bzw. Einbezug im Schulalltag [summary } \\
\text { of knowledge from literature on the topic of learning and description of } \\
\text { applications in schools] }\end{array}$ \\
\hline Research paper & - Research Paper \\
\hline Essay & $\begin{array}{l}\text { - Beschreibung eigener berufsspezifischer Reflexionen bzw. } \\
\text { Stellungnahmen zu einer Fragestellung auf der Grundlage von } \\
\text { Fachliteratur zum Thema (Umfang je nach Aufgabenstellung: } 1-2 \text { Seiten) } \\
\text { [description of personal job-related reflections or a research question } \\
\text { based on literature in the field (size depending on the requirements: } 1-2 \\
\text { pages)] } \\
\text { - } 3-4 \text { seitige schriftliche Arbeit über ein Thema, meist nicht empirisch, aber } \\
\text { literaturgestützt [3-4 page written paper on a topic, generally not } \\
\text { empirical but literature-supported] }\end{array}$ \\
\hline Proposal & $\begin{array}{l}\text { - Projektskizzen/ Protokolle des Arbeitsprozesses [project drafts / notes on } \\
\text { the work processes] }\end{array}$ \\
\hline
\end{tabular}

Tables 1-3 are documentations of the original questionnaire data and the first classifications used. The higher number of genres mentioned in Italian is due to the higher number of respondents from this university. It may be seen that an exact assignment of genres to categories was not possible. 
Table 2: Genre inventory at HEP-Bejune - first classification (with literal translations).

\begin{tabular}{|c|c|}
\hline Genre family & Listed in survey as: \\
\hline Thesis & $\begin{array}{l}\text { - mémoire de bachelor [bachelor thesis] } \\
\text { - mémoire de maîtrise [master thesis] } \\
\text { - mémoire de master [master thesis] } \\
\text { - mémoire professionnel [thesis, unspecified] }\end{array}$ \\
\hline Lesson plan & $\begin{array}{l}\text { - préparations de leçons [preparations of lessons] } \\
\text { - description d'une situation d'enseignement [description of the teaching } \\
\text { - unituation] } \\
\text { educational sciences] }\end{array}$ \\
\hline $\begin{array}{l}\text { Practical training } \\
\text { report }\end{array}$ & $\begin{array}{l}\text { - rapport d'observation [observation report] } \\
\text { - } \text { rapports, observation des stages [reports, internship observations] } \\
\text { - } \text { travail pratique [practical paper] } \\
\text { - description d'une situation vécue [description of a real life experience] } \\
\text { - bilan d'un module [assessment of a module] }\end{array}$ \\
\hline Reflective text & $\begin{array}{l}\text { - réflexions spontanées [spontaneous reflections] } \\
\text { - } \text { ceprésentations [representations] } \\
\text { - journal de bord [journal] }\end{array}$ \\
\hline Seminar paper & $\begin{array}{l}\text { - des travaux d'analyses de données [data analysis papers] } \\
\text { - analyse d'article, de textes sur des thèmes [article, text and theme anaysis] } \\
\text { - prise de position sur une problématique [written comment on a topic] } \\
\text { - séminaire [seminar] } \\
\text { - texte scientifique [scientific text] } \\
\text { - travail de recherche [research paper] }\end{array}$ \\
\hline Summary & $\begin{array}{l}\text { - rédaction de résumé des textes [summary writing] } \\
\text { - synthèse / compte-rendu de lecture [synthesis / minutes on readings] } \\
\text { - synthèse de textes argumentée [synthesis on argumentative texts] } \\
\text { - résumé [summary] }\end{array}$ \\
\hline Minutes or notes & $\begin{array}{l}\text { - prise de notes [note taking] } \\
\text { recorded conversation] }\end{array}$ \\
\hline Essay & $\begin{array}{l}\text { - préparation d'exposés brefs, essais variés, texte argumentatif [preparation } \\
\text { of short compositions, various essays, argumentative text] } \\
\text { - travaux de paper/essay] } \\
\text { - dissertation [essay] }\end{array}$ \\
\hline Exam & - contrôle de connaissances [assessment of knowledge] \\
\hline
\end{tabular}

In the first step, we had to work with guesses and inferences. In some instances, similar or identical genres appear as part of several categories, though with a different name. The first genre inventories were seen as a base for discussion with insiders only. All in all, however, a number of similar genre families appeared in all three universities. 
Table 3: Genre inventory at SUPSI - first classification (with literal translations).

\begin{tabular}{|c|c|}
\hline Genre family & Listed in survey as: \\
\hline Thesis & $\begin{array}{l}\text { - lavoro di diploma [diploma paper] } \\
\text { - lavoro di diploma del bachelor [bachelor diploma paper] } \\
\text { - tesi di diploma [diploma thesis] } \\
\text { - tesi [thesis] } \\
\text { - } \text { progetto di lavoro di diploma [diploma paper project] } \\
\text { - progetto di ricerca per il lavoro di diploma [diploma paper research project] }\end{array}$ \\
\hline Lesson plan & 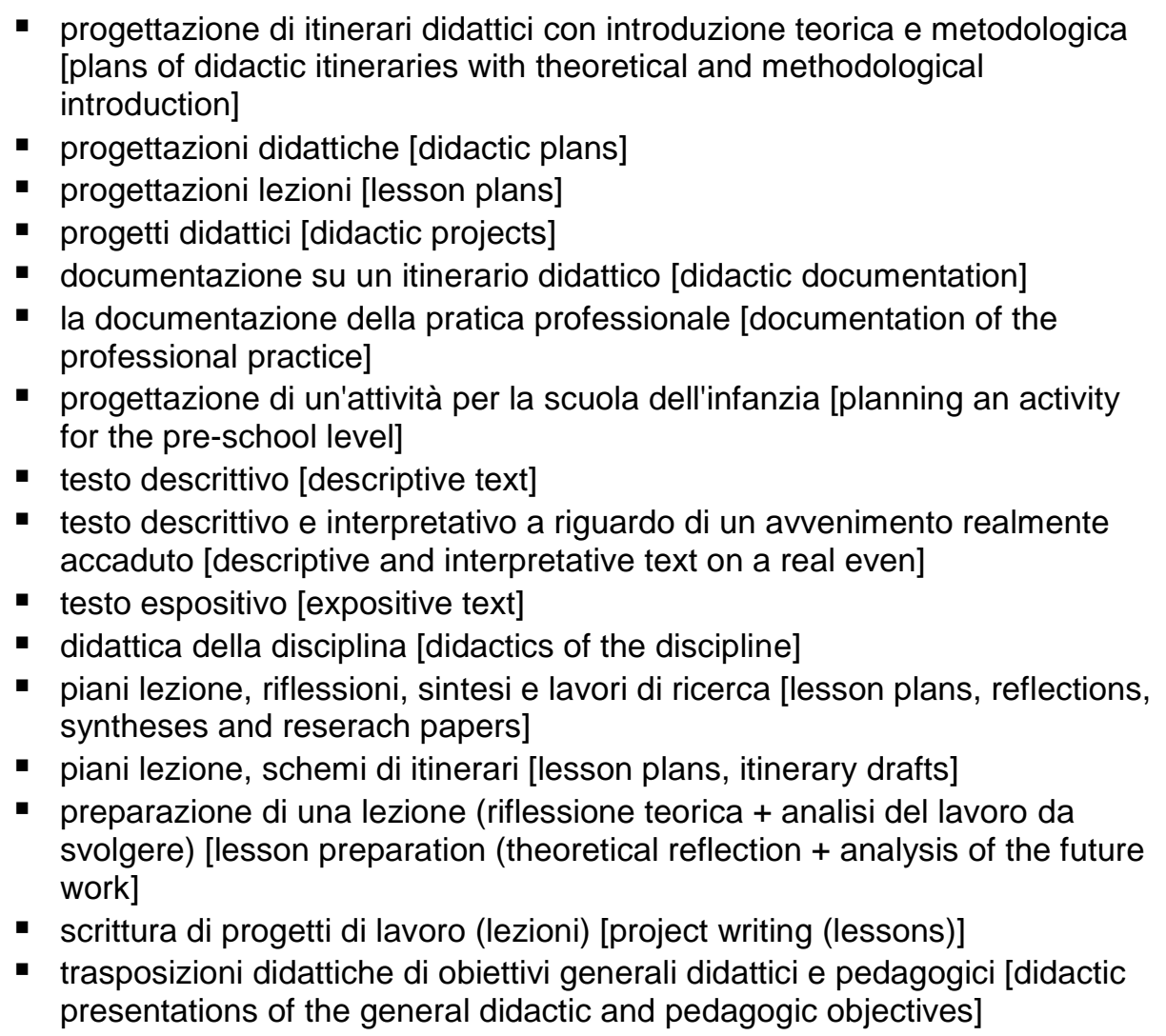 \\
\hline $\begin{array}{l}\text { Practical training } \\
\text { report }\end{array}$ & $\begin{array}{l}\text { - documentazione visita pratica professionale [documentation on the } \\
\text { - } \text { documentazioni della pratica professionale (dossier) [documentation of the } \\
\text { professional practice (file)] } \\
\text { - dossier pratico per un itinerario relativo ad una classe di scuola elementare } \\
\text { [practical file on a elementary school lesson] } \\
\text { - dossier sulle pratiche professionali [file on professional practice] } \\
\text { - rapporto su un colloquio [report on an intervies] } \\
\text { - resoconto di una lezione [report on a lesson] } \\
\text { - } \text { bilanci legati alle pratiche professionali [conclusions on professional } \\
\text { - } \text { dractice] } \\
\text { - } \text { documentazioni relative alle pratiche professionali (tirocinio) } \\
\text { - presentazioni di percorsi esperenziali [presentations on experiential paths] } \\
\text { - rapporti di ricerca [reserach reports] } \\
\text { - testi di sintesi, bilanci di attività, risposta ad interrogativi [synthesis texts, } \\
\text { statements of activity, responses to questions] }\end{array}$ \\
\hline
\end{tabular}




\begin{tabular}{|c|c|}
\hline Reflective text & $\begin{array}{l}\text { - journal de bord } \\
\text { - riflessione personale [personal reflections] } \\
\text { - diari di pratica professionale [diaries of professional practice] } \\
\text { - narrazioni biografiche [biopgraphic narrations] } \\
\text { - riflessione sul proprio operato a seguito di un periodo di tirocinio di } \\
\text { insegnamento o di singole lezioni svolte [reflection on the personal } \\
\text { development after the period of professional training and the organisation } \\
\text { of lessons] } \\
\text { - riflessioni critiche [critical reflections] } \\
\text { - riflessioni critiche in merito agli argomenti affrontati durante le lezioni } \\
\text { - } \text { rifitical reflections regarding the topics approached during lessons] } \\
\text { behaviour or on the students' behaviour] } \\
\text { - riflessioni sulle attività di tirocinio [reflections on training activities] } \\
\text { - scrittura auto-biografica [auto-biographical writing] } \\
\text { - testi di pratica riflessiva (bilanci, riflessioni personali) [texts of practical } \\
\text { reflections (conclusions, personal reflections)] }\end{array}$ \\
\hline Seminar paper & $\begin{array}{l}\text { - } \text { progettazione di ricerche [research plans] } \\
\text { - } \text { ricerca bibliografica [bibliographic research] } \\
\text { - } \text { ricerca documentale [documenting research] } \\
\text { - } \quad \text { ricerca documentale nell'ambito della ricerca in educazione [documenting } \\
\text { research in the context of educational research] } \\
\text { - } \quad \text { ricerca in letteratura [literature research] } \\
\text { - } \text { ricerca sulle fonti [resource research] } \\
\text { - } \text { testo critico e di ricerca intorno al tema dei riconoscimenti [critical and } \\
\text { reserach text on the topic of awards] } \\
\text { - } \quad \text { analisi di casi o di situazione-problema [case analysis on problem } \\
\text { - } \text { situations] } \\
\text { - } \text { biografie di apprendimento [learning biographies] } \\
\text { - } \text { presentazione di ricerche di gruppo [group research presentations] } \\
\text { - presentazione di ricerche individuali [individual research presentations] } \\
\text { - presentazione sintetica di quanto proposto in pratica e riflessione critica a } \\
\text { posteriori [synthetic presentations of lesson plans and follow-up critical } \\
\text { - } \text { preflection] }\end{array}$ \\
\hline Abstract & $\begin{array}{l}\text { - brevi testi di sintesi [abstracts] } \\
\text { images and films] } \\
\text { - schede di sintesi che prevedono delle classificazioni concettuali [synthesis } \\
\text { containing conceptual classifications] } \\
\text { - mappe concettuali riassuntive [summarizing conceptual maps] } \\
\text { - schemi e sintesi della lezione svolta da loro [drafts and syntheses of } \\
\text { lessons organized by students] } \\
\text { - schemi, mappe concettuali, mappe didattiche [drafts, conceptual maps, } \\
\text { - } \text { didactic maps] } \\
\text { sintesi di un articolo scientifico [summary of a scientific article] }\end{array}$ \\
\hline Minutes & - appunti [notes] \\
\hline Essay & - essay \\
\hline
\end{tabular}




\begin{tabular}{|c|c|}
\hline Exam paper & $\begin{array}{l}\text { - tema di italiano [essay on the Italian language] } \\
\text { - } \text { reflective essays: students discuss the relevance of the theory for their } \\
\text { practice } \\
\text { - testi argomentativi (risposte a domande aperte durante gli esami) } \\
\text { - } \text { testi espositivi [infomative texts] } \\
\text { - testi informativi o argomentativi [informative and argumentative texts] } \\
\text { - testi narrativi [narrative texts] } \\
\text { - testo argomentativo/espositivo da elaborare a casa } \\
\text { [argumentative/informative text to be written at home] } \\
\text { - esame scritto [written exam] } \\
\text { - verifica di italiano [exam in Italian language] } \\
\text { - esame finale scritto [written final exam] }\end{array}$ \\
\hline
\end{tabular}

It also became obvious that there were some typical genres or genre families for some universities. For the Italian-speaking university, written exams ('esame scritto') seem to be an important issue while in the French- and German-speaking universities such a writing practice has been mentioned only once. Genres like the 'testo descrittivo' or 'testo espositivo' we would expect in the context of secondary rather than in tertiary education.

\subsection{Classification of genres}

The preliminary categorizations described in the previous section served the function of reducing the high numbers of genres to a manageable number which could be discussed with members of the three universities. At PH Zurich, this discussion led to a new list of genre families where genres were grouped differently (Table 4). Some texts were eliminated if they could not be identified as a genre by our informants while others were added (e.g. the 'didactic thesis'), when they were considered to be essential. The portfolio, for example, proved to be part of the final exam and thus had a thesis-like status. It represents as we were told, a collection of major texts to be chosen from all assignments during study time complemented by additional reflections. Another form of thesis in the Germanspeaking university is called in-depth study' ('Vertiefungsarbeit') - a term that was not familiar to us, causing us to miss it in the first classification attempt. Next to the Portfolio and the in-depth study' there is a third thesis, called 'didactic thesis' (see below) in which a topic from teaching is elaborated. By simply looking at their names, the significance of these three thesis genres could not have been decoded.

As we learned from the faculty workshop, writing about teaching proved to be a core element of writing practices and was represented by several genres which we divided in two different genre families: lesson plans and practice reports (Table 4). Planning lessons (for school teaching) is a significant preparation for the professional role of teachers and demands the integration of didactic with subjectspecific and curricular knowledge. The practice reports, in contrast, though also connected with preparing students for their professional role, were characterized by their documentary and reconstructive nature in recounting own teaching experiences which was to be connected with personal reflections. Reflections seem to play a greater role than was assumed from the questionnaire data and it seems justified to include an additional genre family of 'reflective texts' to account for genres like learning journals, blogs, and personal reflections. Our interview partners from all three universities, but especially those from $\mathrm{PH}$ Zurich, considered reflection as the core element of student learning activities. In their eyes, all theoretical and methodological knowledge has to pass through personal reflection. 
Table 4: Genre families art PH Zurich after faculty workshop

- Final Thesis: Portfolio, Präsentationsportfolio, Vertiefungsarbeit, Berufspraktische Prüfung, Bachelorarbeit, Didaktische Abschlussarbeit, Master Thesis

- Research reports: Fallstudien, Projektskizzen, Research

- Seminar papers: Fachwissenschaftliche Hausarbeit, Miniforschungsarbeiten, Literaturarbeit, Wissenschaftliche Texte, Zusammenfassen des Hintergrundwissens, Medienprojekte

- Lesson plans: Kommentierte Unterrichtsplanung, Didaktische Analysen, Lernprozessdokumentationen, Planung von Lektionen, Unterrichtsplanung (mit Begründung, Material), Unterrichtsprojekte

- Practical training reports: Falldokumentationen mit Kommentaren (kleinere Aufträge), Praxisbericht

- Reflective texts: Learning Journal, Persönliche Reflexion, Reflexive Texte

- Essay/Blog/Webtext: Blogeinträge, Webquesteinträge

- Notes

- Other: Zusammenfassungen, kritische Diskussionen, Anträge und Gesuche, kreative Texte, Arbeitsstrategien

Seminar papers and research reports are, in a similar way, two different components of one cluster which in this case serves the purpose of research-oriented learning. While the seminar paper is more related to the acquisition of theoretical knowledge, the writing of a research report is connected with a clearly defined research project. There were several other texts or genres mentioned occasionally (like 'critical discussion', 'proposals' and 'creative writings') which we did not include in the overall genre inventory as our informants would not assign them a major role in their department's writing culture.

We were able to identify similar categories from the workshop at SUPSI in Locarno and the interviews at HEP-BEJUNE. This enabled us to draw a first version of the genre mapping of the three Swiss universities.

\section{Contrastive Genre Mapping}

\subsection{Overview}

What connects the writing cultures of all three universities, is the high importance given to the professional and educational texts (such as lesson plans and practical reports) as well as to the reflective texts that are required in different variations, which altogether seem to be the genre families typical for teacher education.

The high importance of professional and reflective texts, however, leads to a split in the genre world of the UTEs between the genres of 'academic writing' usually required at universities (seminar papers, summaries of articles, research reports, etc.) and the reflective and professional genres. Both genre groups follow different conventions and require different writing skills. While the university context demands the connection to research (academic genres) the context of professional teaching demands that knowledge becomes personal in order to be useful for practice (reflective and professional genres). This split is common to all three universities.

Figure 1 gives an overview of the genre families common to all three universities (grey fields) and of those we found only at specific universities (other fields). In the following sections we summarize what seems special about the genres and genre families mentioned in this diagram. 


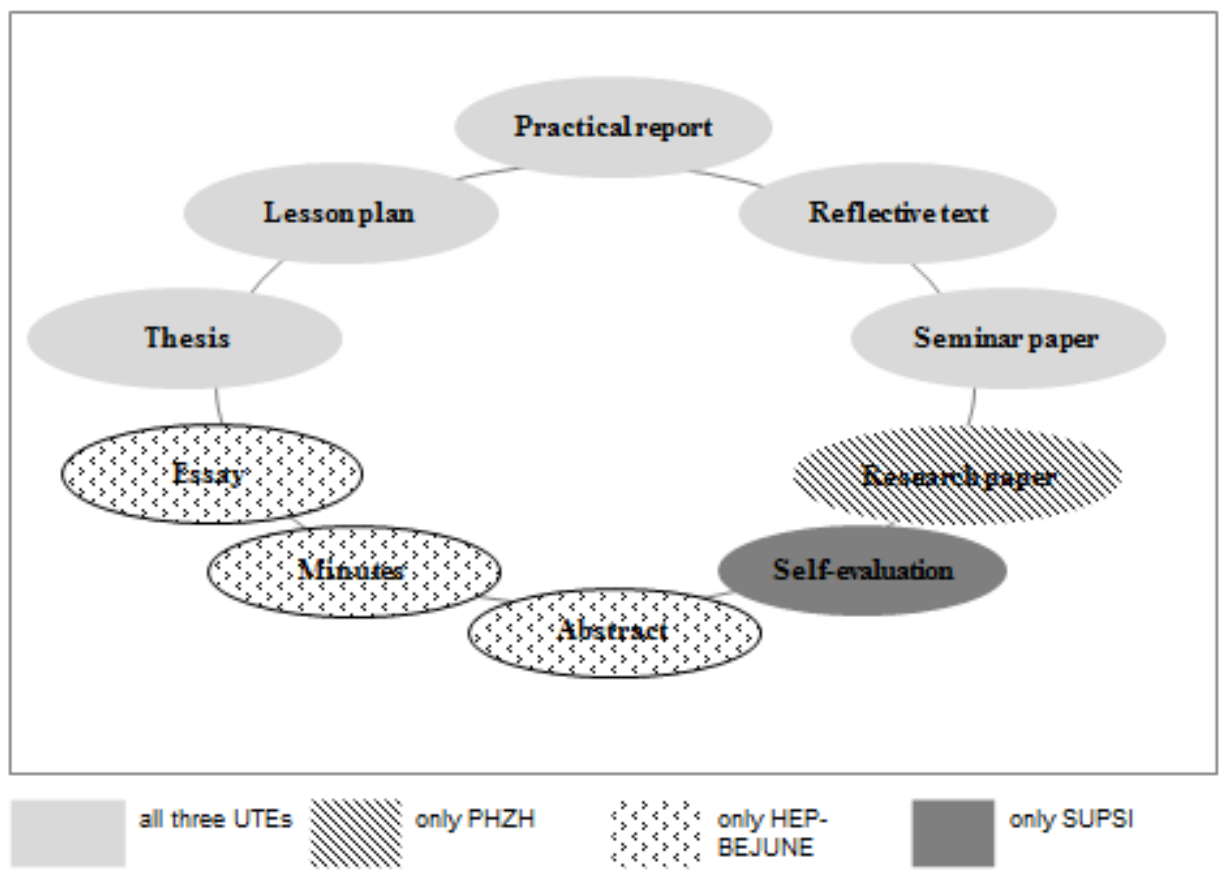

Figure 1: Common and specific genre families at the three UTEs

\subsection{Theses}

Theses are required at all three universities, but not in the same form. Here it is the PH Zurich that makes the exception. Instead of a typical final thesis, three entirely different genres are required. The first is called in depth study' ('Vertiefungsarbeit') and resembles the more traditional type of thesis, usually a theoretical or empirical paper on a narrowly delineated topic. The second is called 'documentation for the practical examination' which we referred to as 'didactic thesis' earlier, and is composed of several texts: a process planning text, presentation, documentation and evaluation of a teaching project. Such theses have up to 60 pages and there are relatively strict guidelines for their composition and evaluation. The third genre is a 'presentation portfolio' that is written during the entire study time and is supposed to document and reflect it. Students write the portfolio under the guidance of mentors, who accompany their pedagogical training. Students are free to choose which papers they add to their portfolio but have to reflect each one. They may also add texts which they have read not written. The portfolio will be graded. No other university requires such a course-related portfolio, although portfolios are used in individual courses at SUPSI.

At the French UTE, the thesis is called 'mémoire' ('mémoire de bachelor', 'mémoire de maitrise', 'mémoire de master', 'mémoire professionnel'). Instead of 'mémoire', the term 'travail' is also used. The French thesis is a research related paper. Competences for research writing are built in several preparatory courses on topics like summaries of research articles, citations or stylistic exercises. At SUPSI, for theses the terms 'tesi', 'lavoro di diploma', or 'progetto di lavoro di diploma' are used. The thesis is to be aligned either with theoretical or empirical work.

\subsection{Lesson plans}

Lesson plan texts and lesson description texts take the shape of two major genres with respect to writing practices which are specific for writing at UTEs. The core pedagogical genres are lesson plans and practical training reports. At the German UTE, for lesson plans', we found various names and name variations: annotated lesson plan, pedagogical plans including lesson plans and preparations, teaching projects, learning process documentations, lesson plan with motivation and materials etc. The planning and processing of educational steps is connected with theoretical and pedagogical knowledge as well as with reflections. Similar text types can be found at the other two universities. The number of entries in the HEP-Bejune questionnaire is lower, but we cannot say to what extent the 
lower number of answers reflects the lower number of informants or the (un-) importance of this genre.

Professional writing on vocational or professional experiences, as in internships is, in any case, a central point in the text practices of pedagogical study programmes. In Italian, informants give examples such as 'documentazioni relative alle pratiche professionali (tirocinio)', and in French 'une restitution de stage pratique' were mentioned.

\subsection{Seminar papers}

The seminar paper is a typical German-language genre, but it has found its place in higher education contexts in many other countries as well. In this context it is not remarkable that the seminar paper is only mentioned at the German-speaking UTE, whereas the French-speaking informants mention 'seminar' but not 'seminar paper'. It can be assumed, however, that there are similar writing practices like term papers but which are named differently. On the other hand, it is rather difficult to distinguish between seminar papers and research papers. In contrast to the seminar papers, in which theoretical aspects are dominant, the presentation of the empirical process is what counts in a research paper. At all three universities, there are special research courses which are connected with different writing requirements. At PH Zurich, there is a compulsory research course in which the paper to be written is called 'research paper'. At HEP-Bejune, on the other hand, there are several mandatory preparation courses for the bachelor thesis but only the writing of summaries of research articles and research findings is required, not a complete research paper. The genres mentioned at HEP-Bejune, which may come close to what the seminar paper is for the German-speaking university, are the 'travaux d'analyse', the 'prise de position sur une problématique' or the 'texte scientifique'. It must be left to a more text-based study to find out about the true nature of these genres and their similarity to a seminar paper.

\subsection{Research reports}

The term 'ricerca' is used at SUPSI for research-related papers but it cannot be accurately distinguished from research and term papers. More frequently than in the other two universities, oral presentations in research contexts are mentioned, suggesting either familiarity with seminar practices or a predominance of oral practices over writing, as in Italian universities. Similar to the situation at HEPBejune, genre names are associated with specific methodological issues, such as 'ricerca bibliographica', 'ricerca documentale', 'ricerca sulle fonti', which actually refer to research processes, not to papers.

\subsection{Essays}

The essay is probably the most controversial genre mentioned in the questionnaires. The term has different connotations in all three languages and it can probably be defined only by referring to its length (or rather, brevity) and its argumentative nature. At PH Zürich, an answer from the survey was: '3-4 page written paper on a topic which is usually not empirical, but literature-based,' which may be identified as the description of an essay, but was not explicitly called so. At PH Zurich, essays seem to be used as assignments from individual teachers but are not an established genre. At the HEPBejune, the dissertation was mentioned several times, which is a popular essay form in French schools and universities training academic discourse. Other entries describe fairly accurately what dissertation-like essays might be: 'préparation d'exposés brefs', 'essais variés', 'texte argumentative' [preparation of short texts, various essays, argumentative texts]. At the Italian-speaking university, the term 'essay' was used twice but other paraphrases were preferred: 'testi argomentativi', 'text espositivi', 'testi informativi o argomentativi, testi narrativi'. Several references also indicate that essays are often used for evaluation purposes. Unlike in the English-speaking countries, the essay does not seem to dominate the writing culture of any of the three universities but has its place at least in the French and Italian speaking universities.

\subsection{Reflective texts}

The variety of reflective texts is remarkable. All three institutions use logs, journals and writing or learning diaries ('journal de bord', 'carnet de bord', 'diari di pratica professionale). Reflective texts are often associated with writing about professional experience. 
At all three universities, reflective genres are used in connection with class activities (e.g. reflecting on lectures or mentoring experiences), in connection with learning of disciplinary knowledge, and probably most often - in connection with the preparation and retrospective evaluation of teaching experiences. SUPSI is the only case where 'critical reflection' is mentioned indicating that there is a fluid transition from reflection to critical thinking. The term 'bilancio' (evaluation) seems to be used synonymously at SUPSI.

\subsection{Specific genres}

At each of the three universities we found specific genres which were not used by the others. Table 5 shows the ones we identified.

Table 5: Specific genres at the three UTEs

\begin{tabular}{|c|c|c|}
\hline \begin{tabular}{ll}
\multicolumn{1}{c}{ PHZH } \\
- $\quad \begin{array}{l}\text { Vertiefungsarbeit [in- } \\
\text { depth study] }\end{array}$ \\
- & Medienprojekte \\
[media projects] \\
- Blogeinträge, \\
Webquesteinträge \\
[blog and WebQuest \\
entrances]
\end{tabular} & $\begin{array}{l}\text { HEP-BEJUNE } \\
\text { Manipulations, trans- } \\
\text { formations de phrases } \\
\text { complexes ou de tex- } \\
\text { tes [manipulations, } \\
\text { transformations of } \\
\text { complex phrses or of } \\
\text { texts] } \\
\text { Rédaction de réponses } \\
\text { à des questions po- } \\
\text { sées sur des textes } \\
\text { aussi bien oraux } \\
\text { qu'écrits de tous gen- } \\
\text { res [written responses } \\
\text { to questions on oral } \\
\text { and written texts of all } \\
\text { genres] } \\
\text { Ecriture clinique [clini- } \\
\text { cal writing] }\end{array}$ & \begin{tabular}{ll}
\multicolumn{1}{c}{ SUPSI } \\
- \\
Rielaborazione [re- \\
elaboration] \\
- $\begin{array}{l}\text { Riflessioni critiche } \\
\text { [critical reflections] }\end{array}$ \\
- Presentazioni di \\
perscorsi esperenziali \\
[presentations of \\
experiential paths] \\
- Autovalutazioni rispetto \\
al profilo di competenze \\
dell'insegnante [self- \\
evaluation regarding the \\
teacher's competences]
\end{tabular} \\
\hline
\end{tabular}

For $\mathrm{PH}$ Zurich, it is characteristic, as previously shown, that several final papers are required, including a portfolio, an in-depth study' and a didactic paper. Subject-specific papers such as 'media projects' and WebQuest tasks' are also exclusively used at the PH Zurich but seem to be tied to a special disciplinary context (Media Studies).

At HEP-Bejune, there are multiple nominations for writing practices that relate to summarizing and reformulation practices ('manipulations', 'transformations de phrases complexes ou de textes') or to answers to examination questions.

An unusual form of mixture between learning and writing is called 'rielaborare' at SUPSI. Students must reflect retrospectively on the knowledge they have acquired in a course or module. It is a blend of recapitulation, reflection and memorizing aid, which is not used in this form in any of the other two universities. During the interviews, it became clear that this is not a marginal didactic tool, but a core element of the learning and teaching philosophy of the institution.

Both, in the French- and the Italian-speaking university, reflective genres were mentioned and seem to be dedicated to the quest for self-awareness, such as the 'narrazioni biographiche' (biographical narrative), or the 'riflessioni personali' (personal reflections) at SUPSI, as well as 'réflexions spontanées' (spontaneous reflections) and 'ecriture clinique' (clinical writing), representing a psychoanalytically inspired experiential writing form with reference to Cifali (1999). 


\section{Discussion}

In this paper, contrastive genre mapping has been used to explore the textual worlds of three comparable Swiss universities from different language regions. As genre and writing terminologies differ considerably from culture to culture, we provided translations for the genres from each of the three universities. We used our study as a step to widening our conceptual thinking beyond the cultural imprinting that affects research and teaching in writing.

What surprised us most about the results is the correspondence of the three universities with respect to the dominant genre families. What has proved to be stronger than the influence of the language on genre, is the influence of the discipline and of the practice requirements for teacher education. The dominance of reflective and didactic genres as a counterpoint to 'pure' academic writing seems common to all three universities. We cannot tell at the moment, how much this correspondence is a result of the coordination of teachers' education within Switzerland or rather a unique feature of teacher education that applies to other countries as well.

As far as the differences between the studied genre profiles of the universities and their neighboring countries are concerned, it seems as if their connection to a Swiss writing culture is higher than to the German, French and Italian background cultures. In Italy, teaching and learning cultures are orally dominated and writing plays a minor role in teaching (Dell'Aversano and Grilli 2009). The SUPSI students, however, write no less than their fellow students in the French and German part of Switzerland. The writing culture at the German speaking university seems well connected to the typical seminar writing tradition with its emphasis on conventionalized reproduction of sources, but nonetheless includes personal and professional writing with equal weight as in the two other universities.

A kind of difference in the three writing cultures that emerged slowly in our analysis (and needs more backing by research) is the relation between (academic) knowledge and the personal sphere. At the Italian speaking university we found a strong quest for expressing academic knowledge in a personal voice. Knowledge is supposed to be integrated in personal value systems and student writings have to reflect the personal effort to make sense of knowledge. At the French speaking university, in contrast, students have to learn to use the voice of the discursive genres of their discipline. The personal should not appear in the text but should be expressed as part of the discursive presentation. The German speaking university, in turn, seems to demand a balanced act in which students have to practice the switch between the academic and the personal by using the respective genres. 


\section{References}

Bazerman, C. (1994). 'Systems of genres and the enactment of social intentions'. In: F. Aviva and M. Peter (eds.): Genre and the New Rhetoric. London: Taylor and Francis, 79-101.

Bhatia, V. K. (2002). 'Applied genre analysis: a multi-perspective model'. Ibérica: Revista de la Asociación Europea de Lenguas para Fines Específicos (AELFE) 4, 3-19.

Chitez, M. and Kruse, O. 2012. 'Writing Cultures and Genres in European Higher Education'. In: M. Castelló and C. Donahue (eds.): University Writing: Selves and Texts in Academic Societies. Studies in Writing Series, Vol. 24. Emerald Group, 151-175.

Cifali, M. (1999). 'Un rapport de la psychanalyse aux sciences de l'education'. Raisons educatives 1 , 293-313.

Connor, U., Nagelhout, E., and Rozycki, W. V. (eds.). (2008). Contrastive Rhetoric: Reaching to Intercultural Rhetoric. Amsterdam: John Benjamins.

Custance, B. (2006). 'Whole-school genre maps: a case study in South Australia'. In: R. Whittaker, M. O'Donnell and A. McCabe (eds.) Language and Literacy: Functional Approaches. London: Continuum, 144-158.

Foster, D., and Russell, D. R. (2002). Writing and learning in cross-national perspective: Transitions from secondary to higher education. New Jersey: Lawrence Erlbaum.

Martin, J. R. and Rose, D. (2008). Genre Relations: Mapping Culture. London: Equinox.

Nesi, H., and Gardner, S. (2012). Genres across the Disciplines: Student writing in higher education. Cambridge: Cambridge University Press.

Nesi, H., Gardner, S., Thompson, P., Wickens, P., Forsyth, R., Heuboeck, A., Holmes, J., Hindle, D., Ebeling, S., Leedham, M., and Alsop, S. (2008). An Investigation of Genres of Assessed Writing in British Higher Education: Full Research Report. ESRC End of Award Report, RES-00023-0800. Swindon: ESRC.

Swales, J. M. (1990). Genre Analysis: English in Academic and Research Settings. Cambridge: Cambridge University Press. 